\title{
Formación de una cultura científica a través de tareas para El aprendizaje
}

DOI: $10.46932 / \mathrm{sfjdv2n2-123}$

Received in: March 1st, 2021

Accepted in: May 30th, 2021

\section{Dr. Jorge Luis Contreras Vidal}

Profesor-investigador de la Universidad Central "Marta Abreu" de Las Villas (UCLV), Sede "Félix Varela".

'Cuba Carretera a Camajuaní Km. 5 y 1/2. Santa Clara. Villa Clara. Cuba.

E-mail: jcontreras@uclv.cu

\section{Lic. Yanay Hidalgo Leonard}

Docente del Instituto Preuniversitario "Capitán Roberto Rodríguez” (IPUCRR). E. Villa Clara. Cuba.

E-mail: yanayhl@crr.sc.vc.rimed.cu.

\section{MSc. Loor Almeida Rafael Antonio}

Docente de la Universidad Laica Eloy Alfaro de Manabí (ULEAM) Extensión en El Carmen.. https://orcid.org/0000-0002-5392-6734. Ave 3 de julio y Carlos Alberto Aray, El Carmen Manabí, Ecuador.

E-mail: antonilooralmeida@ hotmail.com

\section{MSc. Henry Curbelo Sosa}

Docente de la Universidad Mundo Maya (UMMA), Campus Campeche.

. Calle 10 número 17. Poblado IMI II. San Francisco de Campeche. Campeche. México.

E-mail: henrycurbelo@umma.com.mx.

\section{MSc. Xenia Pedraza González}

Profesora-investigadora del Centro de Gestión Internacional de Capacitación y Posgrado (GESICAP S.A). Ave 3 de julio y Calle 24 de julio, El Carmen, Manabí, Ecuador.

E-mail: xeniapedraza@gmail.com

\section{RESUMEN}

El presente artículo versa sobre la experiencia docente en la aplicación de tareas de aprendizaje a los estudiantes que estudian en la UMMA, en la UCLV, en la ULEAM y en el IPUCRR, tanto en especialidades de ciencias como en las ingenierías, con vistas a generar en los mismos, a través de una educación científica, una cultura científica de calidad. La falta de motivación hacia el estudio en determinadas asignaturas de las especialidades mencionadas y de una cultura general y científica por parte de los estudiantes, conllevó a confeccionar y colectar las tareas de aprendizaje, como una de las alternativas para resolver la problemática planteada. Durante los cursos en las que se han aplicado las mismas, se han obtenido resultados satisfactorios en cuanto a lograr sembrar la semilla de la motivación y se ha ampliado la cultura científica en los estudiantes.

Palabras Clave: cultura científica, educación científica, tareas de aprendizaje. 


\section{INTRODUCCIÓN}

En la actualidad uno de los retos de la educación es proporcionar una educación científica a todos los ciudadanos, que perciban la ciencia como una actividad cultural que contribuya a prepararlos para la vida, como una de las premisas para la educación permanente. La educación científica del individuo lo ha de conducir "no sólo a saber de ciencia, sino también sobre la ciencia” (Núñez, J., 1998): sus aspectos culturales, epistemológicos, éticos, sus relaciones con la tecnología y su repercusión social.

Hoy día se vive una época por demás globalizada en la que se producen una demoledora cantidad de conocimientos y existe un inmenso desarrollo de la ciencia y de la tecnología. Sus resultados impactan en los cambios que se suceden en todas las esferas de la sociedad y en la vida del ciudadano común.

En este sentido advierte Núñez (1998): “En la perspectiva de los cambios que se suceden en el mundo los países y las personas que no posean una buena formación, susceptible de permanente actualización, quedarán marginadas en lo económico, lo social y lo cultural” (Nuñez, J., 1998, p. 25). Por su parte, Bertucci y Quirolo (2008) consideran que "La imagen tradicional de ciencia y tecnología vertiendo su saber en una sociedad que los recibe y valora positivamente, no se ajusta a la realidad presente; no cabe aceptar que ambas son ajenas a valoraciones, presiones e intereses que caracterizan la vida social" (Bertucci y Quirolo, 2008, p. 147).

La experiencia mundial evidencia que en el nuevo escenario global la información y el conocimiento son una poderosa fuente de riqueza y de progreso sostenible. Sólo los países capaces de hacer del conocimiento, la cultura y el progreso técnico el sustento de la inclusión social, del bienestar colectivo y el motor de la competitividad económica tendrán viabilidad en el concierto mundial. Por ello en el mundo actual se ha convertido el conocer de ciencia en un imperativo, en una necesidad que posibilita que el hombre de hoy día, ayude a convertir a este en un mundo mejor. En la cotidianeidad se presentan constantemente situaciones como la alimentación, la salud, las actividades recreativas, la investigación en las ciencias y la tecnología, entre otras que influyen en los modos de vida y pensamiento y requieren de una decisión, que si no es siempre de connotación científica, al menos necesita del conocimiento elemental de las ciencias para resolverla. Tanto es así, que los avances económicos y sociales, dependen del impetuoso desarrollo de la ciencia y la tecnología; y la producción avanza gracias a los resultados de la actividad científica investigativa.

\section{DESARROLLO}

La ciencia es una esfera de la actividad humana, un fenómeno condicionado por los valores, representaciones, intereses, etc. de los hombres que determinan la dinámica y el curso de su desarrollo y sus producciones. La ciencia se relaciona orgánicamente a la cultura; se penetran y presuponen en su 
interexistencia (Dusú, R., 2003, p. 3). En este mismo sentido, Guadalupe Zamarrón (2006) refiere la tendencia actual de considerar a los ciudadanos partícipes de las decisiones que afectan su vida en todos los ámbitos. Por ello, "...para lograr individuos participativos en esta nueva sociedad, una condición necesaria es el grado de conocimientos y de cultura científica y tecnológica que posean tanto los ciudadanos como los gobernantes, lo cual adquiere enorme importancia en los países en desarrollo, con el fin de disminuir la dependencia global que tenemos con respecto al mundo desarrollado" (Zamarrón, G., 2006, p. 134). En fin, en una sociedad donde la ciencia y la tecnología se hacen presentes de múltiples maneras, la búsqueda de una cultura científica es indispensable para la existencia de una verdadera democracia participativa. Y en una verdadera democracia, los medios de comunicación se constituyen en unos mediadores sociales de primer orden entre los ciudadanos y las instituciones.

De acuerdo a lo anterior, a la hora de escribir los libros de texto de ciencia hay que tomar en consideración la omisión de contenidos metacientíficos. Cabe esperar que la cultura científica de los individuos no solamente contenga los conocimientos básicos de la "ciencia ortodoxa", sino que también incluya aspectos relativos a los riesgos, efectos adversos, usos políticos, dilemas éticos o influencias económicas de la investigación científica y el desarrollo tecnológico, así como la habilidad o capacidad para hacer frente a los objetos tecnológicos de la vida cotidiana.

La finalidad de la ciencia no se ubica por consiguiente, sólo, en la producción de nuevas ideas; en la actualidad se le concibe como un "vehículo cultural de potenciación, adquisición de conocimientos, pensamientos y valores que permite a los futuros ciudadanos percibir tanto las utilidades de la ciencia y la tecnología en la mejora de la calidad de vida como las consecuencias negativas de su desarrollo" (Dusú, R., 2003, p. 3), por lo que es esencial la constitución de bases educativas- desde las estructuras socialmente instituidas, que posibiliten, formalmente, la culturalización científica.

La educación tiene que ser, por lo tanto: natural, científica, integral, desarrolladora, para la vida y con un elevado sentido práctico. Estos son los principios que rigen una educación concebida científicamente. Esta educación se denomina educación científica y es “el proceso continuo y permanente, orientado a la formación y desarrollo de la cultura científica, que contribuya a preparar al hombre para la vida, fundamentalmente con conocimientos científicos vinculados al desarrollo social, de procedimientos y habilidades necesarios para su autoeducación y valores éticos acordes a las necesidades sociales" (UNESCO, 1998, p. 12).

Las universidades y otras instituciones educativas pueden jugar un papel importante en este sentido a través de la elaboración de materiales didácticos, la sensibilización de los profesores y la incorporación en su formación permanente de estrategias, sistemas, modelos, etc. que contribuyan al desarrollo de una 
cultura científica que les permita luego incidir en los estudiantes llevando a cabo un adecuado proceso de educación científica.

Es evidente entonces que se torna imposible educar científicamente, si uno de los más importantes gestores del proceso, el profesor, no posee la cultura científica necesaria como parte de su formación permanente y en correspondencia con los tiempos actuales. Para conseguir esto, se hace vital accionar no solo desde el trabajo metodológico, sino también desde la superación profesional, la extensión universitaria, etc. También hay que estar atentos a las omisiones que existen en los libros de texto o cualquier otro material didáctico que se elabore, porque las mismas van en la dirección totalmente contraria al logro de una educación científica y, por ende, a la formación de una cultura científica de calidad.

El término cultura científica puede ser analizado desde diferentes perspectivas, una de las más extendidas lo conceptualiza como el conjunto "de normas, de valores, jerarquías, criterios de legitimidad" (Dusú, R., 2003, p.3) que se establecen en virtud de una práctica científica. Desde esta acepción se aprecia la cultura científica como una tradición limitada al campo del quehacer científico profesional y, hasta cierto punto, descontextualizada del entorno social. Otra alternativa, no excluyente de la anterior, comprende este término como resultado de la apropiación por el individuo (desde el proceso de enseñanzaaprendizaje) de estrategias, actitudes, conocimientos, valores, etc. relacionados a la práctica científica e investigativa en campos o disciplinas determinados del saber, en aras de una mayor socialización profesional y ciudadana (Dusú, R., 2003).

Según el artículo "La cultura científica, la percepción pública y el caso de la biotecnología” se parte del concepto general de cultura para plantear un concepto de cultura científica: "La cultura científica debe estar relacionada no sólo con la disposición de conocimiento (¿información?) sobre hechos o datos, sino que debe tener en cuenta, reconocer, la importancia de los procedimientos, de los procesos, de la naturaleza del conocimiento en función de los temas y de las técnicas aplicadas. Ante este contexto, parece lógico concluir que la cultura científica es ante todo fruto de la educación” (Muñoz, E., 2002, s.p).

Siendo evidente que en esta definición se considera a la cultura científica como algo más que disponer de conocimiento, sino también de los procedimientos para adquirirlos, así como considerar la cultura científica fruto de la educación, sin embargo, la educación como categoría se da en diferentes contextos en la sociedad, en el caso de la universidad en las comunidades intra y extrauniversitarias, y no en todos ellos la educación es científica. Según Cruces y Vessuri (2004) la cultura científica está dada por “...la comprensión de la dinámica social de la ciencia: quién la produce, hacia qué fines, con qué consecuencias, frente a qué costo de oportunidad, para qué beneficiarios” (Cruces, J. M. y H. Vessuri, 
2004, p. 31), refiriéndose fundamentalmente a la capacidad de la sociedad de tomar decisiones sobre el tema.

Otras definiciones de cultura científica son:

“...la expresión de los valores materiales y espirituales resultados del trabajo científico en su devenir social y que son conservados, reproducidos e incorporados a la propia actividad humana para crear nuevos valores" (Salazar, D., 2004, p. 8). "El proceso y resultado de la educación científica que incluye el conocimiento sobre la ciencia y la tecnología, las habilidades para alcanzarlo y utilizarlo, incidiendo en los modos de actuación ciudadana en los distintos contextos que confirman el grado de humanización alcanzado por el hombre, en correspondencia con los intereses y necesidades de la sociedad en que vive" (Reyes, M., 2006, p.29).

"La comprensión de la dinámica social de la ciencia, de manera que se tejen en una interrelación entre productores de conocimientos científicos y otros grupos sociales, todos ellos como partícipes del devenir de la cultura, produciendo significados cuyos orígenes y justificaciones provienen desde distintas prácticas, intereses, códigos normativos y relaciones de poder, entendiéndose como un devenir continuo" (Vaccarezza, L., 2008, p. 110).

"Cultura científica es la parte de la cultura que posibilita al hombre conformar explicaciones, interpretaciones y predicciones acerca de los fenómenos y procesos, desde lo mejor y más actualizado de la ciencia, a fin de satisfacer necesidades e intereses. Ella incluye habilidades, sentimientos y modos de actuación, dirigidos a interactuar positiva y creadoramente con la naturaleza y la sociedad, lo que le permite asumir conscientemente sus responsabilidades sociales” (Addine, R., 2006, p. 30).

La cultura científica se redefine entonces a la luz de las actuales demandas sociales a través de los siguientes elementos: conocimiento de los conceptos, principios y enfoques teóricos de mayor significación de su ciencia, por lo que no deben omitirse información de ninguna índole; conocimientos acerca de la filosofía de la ciencia y de la lógica de la investigación; saberes teóricos, procedimentales y actitudinales para transferir y generalizar los conocimientos científicos a los problemas de la cotidianidad; habilidades en el manejo de la tecnología y para el proceder metodológico investigativo; conocimientos básicos acerca de la interrelación entre ciencia, tecnología y sociedad; actitud de compromiso social y de intereses relacionados con la ciencia (actitud científica).

La cultura científica, por lo tanto, no solo sitúa a quien la posee en la perspectiva del dominio de los adelantos de una ciencia; en particular; lo forma como un conocedor crítico, reflexivo de la propia historia de la ciencia, de su filosofía apartándole así la posibilidad de una educación más completa y sistematizada; le exige el desarrollo de habilidades y de estrategias para aplicar, creativamente, los saberes 
existentes, hecho que se erige, actualmente, en finalidad del comportamiento cognitivo del individuo y no del ámbito estrictamente profesional.

En consonancia con esta posición, es importante el desarrollo de la actitud científica, a través de la que se crea una disposición estable y activa hacia la búsqueda del saber, la indagación de la realidad, la explicación, la postura de ausencia de conformidad ante lo creado y de recreación de lo existente.

Por lo tanto, la cultura científica, es una resultante de la influencia de diversos factores culturales que determinan la tendencia de la investigación científica y como un resultado de la educación debe propiciar el desarrollo en prospectivo de las capacidades del estudiante acorde con las demandas sociales y profesionales. Una de las vías de facilitar una cultura científica es a través de las Tareas de Aprendizaje.

"El diseño de tareas de aprendizaje para habilidades...integradas impone una muy alta carga cognitiva para los profesores y es una explicación de las dificultades que experimentan en el diseño de tareas de aprendizaje para la educación basada en competencias. Una solución a este problema...en la educación superior, es la capacitación en diseño instruccional y el trabajo colaborativo en pequeños grupos interdisciplinares para el diseño de experiencias educativas que tienen como elemento principal el uso de tareas de aprendizaje diseñadas por los docentes. Dos formas han resultado adecuadas para el desarrollo de estas habilidades en los docentes:

- la capacitación para dar el apoyo cognitivo con un método apropiado para el diseño instruccional

- el diseño de las tareas de forma colaborativa

El diseño instruccional es una función que está y debe estar en manos de los docentes ya que se aprovecha su experiencia académica y profesional, y también permite al docente adaptar las tareas al contexto y antecedente de los estudiantes. Los programas de los cursos sirven como una orientación general dentro del diseño curricular por competencias dejando en libertad al docente para adecuarlo según sus necesidades.... El método de diseño instruccional debe cumplir con las siguientes características:

- permitir enseñar tareas complejas globales con un enfoque integrado

- con tareas de aprendizaje auténticas de la que los expertos encuentran en su vida cotidiana

· coherentes con el diseño curricular por competencias y para el pensamiento complejo $\cdot$ orientado a un proceso de aprendizaje que permita mostrar las competencias y habilidades adquiridas

- permitir la transferencia de los aprendizajes para resolver nuevos problemas

• aceptable y manejable por los docentes, permitiendo aplicar sus propias experiencias y reflexión”. (Verdejo, Pilar y Rosario Freixas, 2009)

A continuación se muestra un conjunto de tareas de aprendizaje, creadas y colectadas por los autores de este trabajo, las que fueron aplicadas a estudiantes de las universidades UMMA, UCLV, ULEAM y del IPUCRR. 


\section{TAREAS DE APRENDIZAJE}

1. Para inflar un balón de fútbol se necesita de una bomba de aire, sí el cuerpo de la bomba fuera de metal se percibe que en el funcionamiento de la misma la bomba se calienta. Explique por qué ocurre esto.

2. Es común percibir que el agua de una garrafa ó tinaja es más fresca que la que se encuentra en una de vidrio. Explica por qué.

3. Una botella de cerveza y una lata también de cerveza permanecen durante un cierto tiempo en el interior de un congelador, este tiempo es suficiente para que ambos recipientes se encuentren a la misma temperatura y en equilibrio térmico con el interior del congelador, sin embargo al retirar los dos recipientes del congelador tenemos la impresión que la lata está más fría que la botella. ¿Cómo usted explica este hecho?

4. Los termos son útiles para conservar bebidas calientes y frías éstos están constituidos por recipientes con paredes dobles de vidrio plateado interna y externamente. En su fabricación se retira todo el aire existente entre las paredes. Explique por qué esas paredes le sirven al termo como aislante térmico.

5. Explique por qué es necesario el uso de gasolina para arrancar los carros que funcionan con alcohol.

6. El circuito eléctrico de un congelador se conecta y se desconecta automáticamente para mantener en su interior una temperatura estable y adecuada ¿Qué dispositivo ejerce ese control de temperatura en los congeladores y cómo es su funcionamiento?

7. ¿Cómo debe ser el desempeño de un motor que suelta chispas fuera de tiempo?

8. ¿Por qué los refrigeradores funcionan mal en los locales en que la temperatura es superior a los $400 \mathrm{C} ?$

9. En general el rendimiento de los motores eléctricos es mayor que el de los motores a gasolina ¿es posible construir un motor térmico a gasolina con mayor rendimiento que uno eléctrico?

10. ¿Cómo se explica que los esquimales construyan sus casas con hielo y logran protegerse del frío?

11. ¿Por qué es necesario el deshielo periódico en los refrigeradores?

12. Describa los procesos energéticos bajo los cuales se llega a producir corriente eléctrica en: a) Una hidroeléctrica. b) Una termoeléctrica. c) Una celda solar. d) Una batería de automóvil. e) Una pila de reloj o mando a distancia. f) Un molino de viento.

13. a) Investigue los diferentes tipos de fuentes energéticas que existen en el planeta Tierra. b) Diga cuál de ellas son renovables y cuáles no lo son. c) Mencione las ventajas y desventajas de cada una de ellas. 
14. Valore el siguiente planteamiento a través de ejemplos concretos:

"El consumo energético tiene un componente apreciable en el uso que se hace de él para producir iluminación. Muchas veces al tomar medidas para ahorrar se producen errores que aumentan el consumo, se disminuye el bienestar visual y se ocasionan daños de tipo permanente a instalaciones $\mathrm{y}$ construcciones".

15. Una de las mejores formas de ahorrar electricidad en cuanto a la iluminación es el aprovechamiento de la luz diurna o luz natural. a) Enuncie un grupo de medidas que usted podría tomar en su casa para aprovechar la luz diurna. b) ¿Qué otras medidas usted podría tomar en su casa para ahorrar electricidad tomando en consideración el color de las paredes, cortinas, etc.?

\section{CONCLUSIONES}

Las tareas de aprendizaje aplicadas a otras esferas del conocimiento son fuente de motivación y de cultura científica, porque para responder las mismas hay que realizar un proceso investigativo que siempre es de interés para el estudiante. Los estudiantes de las universidades UMMA, UCLV, ULEAM y del IPUCRR incrementaron su acervo cultural y científico con la solución a las tareas mencionadas. 


\section{BIBLIOGRAFÍA}

Addine Fernández, R. (2006): "Estrategia didáctica para potenciar la Cultura Científica desde la enseñanza de la Química en el preuniversitario cubano". Tesis en opción al grado científico de Doctor en Ciencias Pedagógicas. Instituto Superior Pedagógico "Enrique José Varona”. Ciudad de la Habana. Cuba.

Bertucci. A. y M. Queirolo (2008): “Alfabetización científica. Responsabilidad social de los científicos". En FECYT. Resúmenes del Congreso Iberoamericano de Ciudadanía y Políticas Públicas de Ciencia y Tecnología. Madrid.

Cruces, J. M. y Vessuri, H. (2004): “Ciencia y Tecnología. Venezolanos participan y opinan”. Primera Encuesta Nacional de Percepción Pública de la Ciencia, Cultura Científica y Participación Ciudadana. Ministerio de Ciencia y Tecnología. Caracas. Venezuela.

Dusú Contreras, Rayda (2003): "Cultura científica: el alcance social de su educación en el contexto universitario”. http://www.uo.edu.cu/ojs/index.php/stgo/article/viewFile/14503345/777

López, J.A. y M. Cámara (2009): "La cultura científica en España”, en El español, lengua para la ciencia y la tecnología: presente y perspectivas de futuro, Inst. Cervantes, Madrid, España.

$\square$ Muñoz E. (2002): "La cultura científica, la percepción pública y el caso de la biotecnología". http://www.iesam.csic.es/doctrab.htm.

Núñez, J. (1998): “La ciencia y la tecnología como procesos sociales. Lo que la educación científica no debería olvidar". Editorial Científico Técnica. La Habana. Cuba.

Reyes Alpízar, Miriam (2007): "La formación de la cultura científica en los pioneros de secundaria básica a través del taller pioneril”. Tesis en opción al grado científico de Doctora en Ciencias Pedagógicas. ISP "Félix Varela". Villa Clara. Cuba.

Salazar, Diana y Fátima Addine (2004): "La interdisciplinariedad y su enfoque sistémico para el trabajo científico en la enseñanza de las ciencias en Interdisciplinariedad: Una aproximación desde la enseñanzaaprendizaje de las ciencias". Editorial Pueblo y Educación. Ciudad de la Habana. Cuba.

UNESCO (2005): “Conferencia Mundial sobre la Educación Superior en el Siglo XXI: visión y acción”. http://www.unesco.org.ve/

Vaccarezza, L. S. (2008): "Exploraciones en torno al concepto de cultura científica". FECYT Resúmenes del Congreso Iberoamericano de Ciudadanía y Políticas Públicas de Ciencia y Tecnología. Madrid. España.

Verdejo, Pilar y Rosario Freixas (2009): "Educación para el pensamiento complejo y competencias: Diseño de tareas y experiencias de aprendizaje". Aseguramiento de la Calidad en la Educación y en el Trabajo, S.C. México Abril de 2009.

Zamarrón, Guadalupe (2006): "De cultura científica y anexas". VVAA Universidad. Universidad de Baja California y Mario Porrúa Edits. México. 